\section{Diagnóstico da doença hepatobiliar na fibrose cística: um desafio}

\section{Caro Editor,}

Gostaríamos de parabenizar os colegas do Hospital das Clínicas da Universidade Federal de Minas Gerais, que vêm, com suas experiências, suscitando discussões relevantes no que diz respeito ao tema fibrose cística $(F C)^{1-7}$.

O artigo publicado sob o título "Validação do escore ultrasonográfico de Williams para o diagnóstico da hepatopatia da fibrose cística" analisou 70 pacientes com FC, dos quais dez foram considerados hepatopatas com base nas alterações bioquímicas e/ou em evidências de hepatoesplenomegalia. Desses apenas cinco apresentavam escore ultra-sonográfico (EUS) > 3 (quatro pacientes com escore entre 4-7 e um paciente com escore 8$)^{1}$.

O escore proposto por Williams et al. em 1995 conseguiu demonstrar boa correlação entre a alteração ultra-sonográfica e as alterações bioquímicas qualitativas e quantitativas de função hepática ${ }^{8}$. Participaram desse estudo 68 adultos, havendo a real necessidade de validação desse método no grupo pediátrico, objetivo do referido artigo. Entretanto, para que os testes de validação sejam realizados, faz-se necessária a busca de um padrão áureo diagnóstico. Nesse sentido, deparamo-nos com a questão: qual é o melhor método para o diagnóstico da hepatopatia da FC: as evidências clínicas de doença hepática ou as alterações bioquímicas?

O consenso norte-americano propõe que a borda hepática de consistência endurecida, na presença de esplenomegalia, é um indicador de envolvimento hepático clinicamente significativo nesses pacientes. Ressalta ainda que, devido à hiperexpansão pulmonar muito freqüente nesses pacientes, se considera a hepatimetria total referência para o diagnóstico de hepatomegalia, no lugar da simples palpação do órgão abaixo do rebordo costal direito. A presença de níveis séricos das enzimas hepáticas (AST, ALT, fosfatase alcalina, GGT) e bilirrubinas acima de 1,5 vez o valor normal de referência por mais de 3-6 meses, excluindo-se outras causas, é indicador de doença hepática9 9 .

Fagundes et al. ${ }^{1}$ consideraram a doença hepática ao avaliar critérios clínicos e bioquímicos. Considerou-se o exame clínico alterado quando se detectou a presença de baço palpável e/ou hepatomegalia, definida como a presença de fígado palpável a mais de $2,5 \mathrm{~cm}$ da reborda costal direita (RCD), de consistência firme. A bioquímica alterada foi definida pelo aumento persistente e significativo $(1,5$ vez o valor normal de referência) de pelo menos duas das enzimas (AST, ALT, fosfatase alcalina, GGT), por um período superior a 6 meses.
Na nossa experiência, as enzimas hepáticas (AST, ALT, fosfatase alcalina, GGT) não foram capazes de identificar quatro entre cinco pacientes com FC e doença hepática avançada, os quais foram submetidos a transplante hepático isolado, e cujo EUS era de $\geq 8$. Isso não nos surpreende, uma vez que os valores normais das enzimas hepáticas são uma constatação que pode ocorrer em pacientes com cirrose compensada e descompensada, sendo comum em pacientes com cirrose biliar ${ }^{9}$.

Utilizamos o escore proposto por Williams et al. ${ }^{8}$ desde $1997^{10}$ e revisamos os exames de 131 pacientes com diagnóstico de FC acompanhados pelo Setor de Pneumologia Infantil do Hospital de Clínicas de Porto Alegre. Com o objetivo de avaliar a sensibilidade e a especificidade das provas bioquímicas, fizemos uma avaliação em sentido oposto àquela realizada por Fagundes et al. ${ }^{1}$. Consideramos que se tratava de doença hepática quando o EUS era $\geq 6$ e procedemos aos testes de sensibilidade, especificidade e valores preditivos positivo e negativo. Os pacientes estudados pelo nosso grupo se assemelhavam em média de idade $(9,3 \pm 4,9$ anos) e prevalência de sexo masculino $(53,4 \%)$ com os descritos por Fagundes et al. ${ }^{1}$. Trinta e quatro (26\%) pacientes apresentaram EUS $\geq 6$. Desses $55,9 \%$ apresentavam algum exame alterado, em comparação com $26,8 \%$ no grupo com EUS $<6(p=0,003)$. Cada exame laboratorial isolado (AST, ALT, fosfatase alcalina, GGT) foi comparado ao resultado do EUS. Observamos uma baixa sensibilidade dos exames laboratoriais para o diagnóstico da hepatopatia da FC ${ }^{11}$.

Qual a diferença entre as duas experiências? Os examinadores das ultra-sonografias eram diferentes? O número de pacientes estudados? A análise da Tabela 4, publicada no artigo em discussão, evidencia um amplo elastério nos intervalos de confiança referentes aos resultados do escore ultra-sonográfico. Isso pode ser explicado pelo número restrito de casos identificados pelos autores, merecendo ser considerado.

Pensamos que a conclusão dos autores, de que o escore ultra-sonográfico é um método pouco sensível para o diagnóstico da hepatopatia por FC, talvez possa ser precoce. Compartilhamos do "desafio" que é o diagnóstico e o tratamento da doença hepatobiliar nesses pacientes. Acreditamos que, atualmente, o diagnóstico precoce desse acometimento reside na soma dos dados clínicos, laboratoriais e ultra-sonográficos.

\section{Renata Gonçalves Rocha}

Pediatra. Mestranda do Programa de Pós-graduação de Pediatria, Faculdade de Medicina, Universidade Federal do Rio Grande do Sul (UFRGS), Porto Alegre, RS

\section{Sandra Maria Gonçalves Vieira}

Doutora em Gastroenterologia. Gastroenterologista pediátrica, Setor de Gastroenterologia, Serviço de Pediatria, Hospital de Clínicas de Porto Alegre (HCPA), Porto Alegre, RS 


\section{Carlos Oscar Kieling}

Mestre em Pediatria. Gastroenterologista pediátrico, Setor de Gastroenterologia, Serviço de Pediatria, HCPA, Porto Alegre, RS

\section{Sabrina Lima Alves}

Aluna da Faculdade de Medicina, UFRGS, Porto Alegre, RS

\section{Sandra Krebs Genro}

Radiologista, Serviço de Radiologia, HCPA, Porto Alegre, RS

\section{Themis Reverbel da Silveira}

Doutora em Genética. Professora adjunta, Universidade Federal do Rio Grande do Sul (UFRGS). Responsável pelo Setor de Gastroenterologia Pediátrica e Transplante Hepático Infantil, HCPA, Porto Alegre, RS

\section{Referências}

1. Fagundes EDT, Silva RAP, Roquete MLV, Penna FJ, Reis FJC, Goulart EMA, et al. Validação do escore ultra-sonográfico de Willians para o diagnóstico da hepatopatia da fibrose cística. J Pediatr (Rio J). 2004;80:380-6.

2. Fagundes EDT, Roquete MLV, Penna FJ, Reis FJC, Duque CG. Triagem diagnóstica da hepatopatia da fibrose cística. J Pediatr (Rio J). 2002; 78:389-96.

3. Oliveira MC, Reis FJ, Oliveira EA, Colosimo EA, Monteiro AP, Penna FJ. Prognostic factors in cystic fibrosis in a single center in Brazil: a survival analysis. Pediatr Pulmonol. 2002;34:3-10.

4. Camargos PA, Guimarães MD, Reis FJ. Prognostic aspects of cystic fibrosis in Brazil. Ann Trop Paediatr. 2000;20:287-91.

5. Reis FJ, Oliveira MC, Pena FJ, Oliveira MG, Oliveira EA, Monteiro AP. Clinical and nutritional aspects in patients with cystic fibrosis: 20 years of follow-up in Clinical Hospital Federal University of Minas Gerais (HC-UFMG). Rev Assoc Med Bras 2000;46:325-30.

6. Reis FJ, Camargos PA, Rocha SF. Survival analysis for cystic fibrosis in Minas Gerais State, Brazil. J Trop Pediatr. 1998;44: 329-31.

7. Macri CN, de Gentile AS, Manterola A, Tomezzoli S, Reis FC, Largo Garcia I, et al. Epidemiology of cystic fibrosis in Latin America: preliminary communication. Pediatr Pulmonol. 1991;10:249-53.

8. Williams SG, Evason JE, Barret N, Hodson ME, Boultbee JE, Westaby D. An ultrasound scoring system for the diagnosis of liver disease in cystic fibrosis. J Hepatol. 1995;22:513-21.

9. Sokol RJ, Durie PR. Recommendations for management of liver and biliary tract disease in cystic fibrosis. Cystic Fibrosis Foundation Hepatobiliary Disease Consensus Group. J Pediatr Gastroenterol Nutr. 1999;28:S1-13.

10. Vieira SMG, Genro SK, Silveira TR. Alterações hepáticas relacionadas à fibrose cística. In: Ferreira $\mathrm{CT}$, Carvalho $\mathrm{E}$, Silva RS, editores. Gastroenterologia e Hepatologia em Pediatria Diagnóstico e Tratamento. $1^{a}$ ed. Rio de Janeiro: MEDSI; 2003. p. 727-40.

11. Alves SL, Rocha RG, Kieling CO, Vieira SMG, Ferreira CT, Genro $\mathrm{SK}$, et al. Relação entre o escore ultra-sonográfico e provas laboratoriais hepáticas para avaliação da doença hepatobiliar nos pacientes com fibrose cística. Rev AMRIGS. 2004; anais do $21^{\circ}$ Congresso AMRIGS:18S.

\section{Resposta dos autores}

Agradecemos o interesse pelo trabalho desenvolvido em nosso serviço e pela possibilidade de compartilhar a experiência dos colegas do Hospital das Clínicas de Porto Alegre em relação à hepatopatia da fibrose cística (FC).
Um dos principais desafios que os estudos sobre hepatopatia da FC enfrentam está na definição dessa afecção, uma vez que não há marcadores sensíveis do envolvimento hepático nem uniformização dos critérios diagnósticos. No entanto, a observação e a experiência junto aos pacientes têm demonstrado que nenhum critério, seja clínico, bioquímico ou ultra-sonográfico, utilizado isoladamente, apresenta sensibilidade e especificidade adequadas para o diagnóstico. Ou seja, as evidências clínicas da doença hepática não constituem um critério melhor para o diagnóstico do que as alterações bioquímicas, ou vice-versa; esses critérios são complementares. Por outro lado, o seguimento longitudinal é importante para a definição dos casos de hepatopatia, que não devem ser rotulados como tal em uma única avaliação, devido ao caráter intermitente não só da bioquímica como também das alterações ultra-sonográficas e do exame físico ${ }^{1}$.

O consenso norte-americano ${ }^{2}$ mantém a recomendação da triagem através do exame clínico e bioquímico regularmente. Embora conheçamos suas limitações, isso justifica a realização desse estudo, utilizando esses critérios como padrão-ouro. A ultra-sonografia abdominal é o teste de imagem mais amplamente utilizado para o diagnóstico do envolvimento hepatobiliar na FC. No entanto, o consenso americano ${ }^{2}$ considera o exame ultra-sonográfico pouco útil na deteç̧ão da doença hepática na $\mathrm{FC}$, porque a esteatose se assemelha à fibrose periportal, ambas muito freqüentes entre os fibrocísticos. Apesar dessas limitações, a ultrasonografia vem sendo cada vez mais utilizada.

É importante ressaltar a dificuldade que existe em se definir hepatomegalia no paciente fibrocístico. De forma geral, o fígado palpável em paciente com doença pulmonar crônica é atribuído ao rebaixamento do diafragma secundário à hiperinsuflação pulmonar. No entanto, a experiência tem mostrado que crianças mais velhas que apresentam hiperinsuflação importante e aumento do diâmetro AP (antero-posterior) geralmente não têm fígado palpável. Acredita-se que o aumento no diâmetro AP contribua para a acomodação do fígado na caixa torácica, diminuindo o efeito do rebaixamento. Alguns autores utilizam a hepatimetria na tentativa de diminuir o erro, apesar da dificuldade da percussão, pela interposição do pulmão secundária à hiperinsuflação. Dessa forma, o consenso ressalta que a textura da borda hepática é clinicamente mais importante do que a hepatimetria. A palpação de fígado abaixo de 2,5 $\mathrm{cm}$ da reborda costal direita (RCD) deve ser considerada anormal em qualquer idade. O rebaixamento do fígado pela doença pulmonar pode ser diferenciado do fígado acometido através das características de consistência e de sua borda. Um fígado palpável abaixo do limite de $2,5 \mathrm{~cm}$, unicamente devido ao rebaixamento pulmonar, deve ser macio e com borda fina e lisa. O baço palpável também deve ser sempre considerado anormal 2,3 .

Em relação aos resultados apresentados pelos colegas, cabe a mesma dúvida em relação ao critério escolhido como padrão-ouro para diagnóstico da hepatopatia da FC. Conforme o consenso americano ${ }^{2}$, as alterações ultra-sonográficas são pouco úteis na detecção e quantificação da fibrose e cirrose no fibrocístico, daí sua limitação como critério diagnóstico quando utilizado isoladamente e, conseqüentemente, 
como padrão-ouro para testar a sensibilidade e a especificidade de outros testes. Como os colegas, encontramos associação entre o escore ultra-sonográfico e as alterações clínicas e/ou bioquímicas. Os pacientes hepatopatas, segundo os nossos critérios, apresentavam escore mais alto do que os não-hepatopatas, mostrando uma associação positiva, assim como os dados de Porto Alegre. No entanto, não consideramos adequado testar sensibilidade e especificidade da bioquímica utilizando apenas o escore ultra-sonográfico como padrão-ouro.

Embora os intervalos de confiança da sensibilidade e especificidade sejam amplos, devido ao pequeno número de hepatopatas identificados em nosso estudo, acreditamos que a maior diferença entre as duas experiências esteja na escolha dos critérios diagnósticos. A triagem da hepatopatia apenas através do exame ultra-sonográfico, como utilizado pelos colegas, fatalmente acarretará em atraso ou subdiagnóstico. Por isso, estamos de acordo com a conclusão de que o diagnóstico da hepatopatia da FC deve se basear na soma dos critérios clínicos, bioquímicos e ultra-sonográficos.

\section{Eleonora D. T. Fagundes}

Mestre em Medicina. Pediatra especialista em Gastroenterologia Pediátrica pela Universidade Federal de Minas Gerais (UFMG), Belo Horizonte, MG

\section{Rogério A. P. Silva}

Médico, Setor de Ultra-sonografia, Hospital das Clínicas, UFMG, Belo Horizonte, MG

\section{Mariza L. V. Roquete}

Professora assistente, Departamento de Pediatria, Faculdade de Medicina, UFMG, Belo Horizonte, MG

\section{Francisco J. Penna}

Professor titular, Departamento de Pediatria, Faculdade de Medicina, UFMG, Belo Horizonte, MG

\section{Francisco J. C. Reis}

Professor assistente, Departamento de Pediatria, Faculdade de Medicina, UFMG, Belo Horizonte, MG

\section{Eugênio M. A. Goulart}

Professor adjunto, Departamento de Pediatria, Faculdade de Medicina, UFMG, Belo Horizonte, MG

\section{Cristiano G. Duque}

Médico formado pela Faculdade de Medicina, UFMG, Belo Horizonte, MG

\section{Referências}

1. Ling SC, Wilkinson AS, Hollmam AS, McColl J, Evans TJ, Paton JY. The evolution of liver disease in cystic fibrosis. Arch Dis Child. 1999;81:129-32.

2. Sokol RJ, Durie PR. Recommendations for management of liver and biliary tract disease in cystic fibrosis. J Pediatr Gastroenterol Nutr. 1999;28 Suppl:S1-13.

3. Colombo C, Apostolo G, Ferrari M, Seia M, Genoni S, Giunta A, et al. Analysis of risk for the development of liver disease associated with cystic fibrosis. J Pediatr. 1994;124:393-9.

\section{Acalasia de esôfago e esofagite eosinofílica}

\section{Caro Editor,}

Gostaria de acrescentar alguns dados em relação à acalasia do esôfago. $\mathrm{O}$ artigo apresentou de forma brilhante a propedêutica diagnóstica e terapêutica para o quadro ${ }^{1}$, mas deixou de informar o dado relacionado ao resultado da biópsia. Trata-se de um dado importante, uma vez que podemos encontrar alterações sugestivas de patologias associadas, que podem estar relacionadas à origem da acalasia esofágica. Como exemplos, vale citar o lupus eritematoso sistêmico, a sarcoidose e a esofagite eosinofílica $(E E)^{2}$.

A EE é uma das causas de dismotilidade esofágica que vem sendo descrita com freqüência cada vez maior, a partir de seu conhecimento e de sua busca pelos médicos ${ }^{3}$. Sua forma de apresentação clínica é muito parecida com o refluxo gastroesofágico, apresentando sintomas como dor abdominal, vômitos e disfagia, entre outros, que não respondem ao tratamento anti-refluxo convencional; além disso, o padrão de pHmetria mostra tendência a altos níveis de $\mathrm{pH}^{4}$. O diagnóstico é feito através da biópsia, que apresenta a presença de eosinófilos no terço inferior esofágico com número acima de 20 ou 24 por campo de aumento de 40 vezes. Em geral, sua continuidade leva a distúrbios de motilidade mais importantes e à apresentação de odinofagia, impactação alimentar, perda de peso, falência no crescimento e caquexia ${ }^{2-4}$.

Apesar de ser mais comumente associada ao espessamento da parede esofágica e sua estenose, a associação entre acalasia esofágica e a esofagite eosinofilica já foi relatada 2,5 . Em uma série de 42 pacientes com diagnóstico de acalasia de esôfago, submetidos à esofagectomia torácica, cujos fragmentos foram estudados histologicamente, 22 pacientes (52\%) apresentavam eosinofilia na camada muscular própria $^{6}$. O papel desses eosinófilos ainda não se encontra bem estabelecido na formação da lesão da acalasia7. Na EE, além dos procedimentos para permitir a passagem dos alimentos, como a dilatação ou cirurgia esofágica, torna-se necessária a investigação relacionada a possíveis alérgenos alimentares, que são encontrados entre 50 a $80 \%$ dos indivíduos afeta$\operatorname{dos}^{8}$. Em geral, a retirada do alérgeno alimentar leva à melhora considerável da sintomatologia; porém, em alguns casos, pode haver persistência ou recorrência do mesmo, sendo necessária a administração de drogas, como corticóides orais ou inalatórios ou, ainda, antileucotrienos ${ }^{9}$. A ausência do encontro de alérgenos alimentares associados também corrobora com o uso dessas drogas ${ }^{8,9}$.

O crescente conhecimento e relato de séries de casos de pacientes com EE, principalmente em crianças, mas também em adultos ${ }^{10}$, motivou a elaboração da carta, sob o aspecto tanto da acalasia quanto da estenose esofágica - ambas associadas a essa doença, que vem adquirindo importância significativa em todo mundo.

\section{Gesmar Rodrigues Silva Segundo}

Mestre em Imunologia pela Universidade Federal de Uberlândia. Médico assistente, Ambulatório de Alergia e Imunologia Pediátrica, Universidade Federal de Uberlândia (UFU), Uberlândia, MG. E-mail: gesmar2@pop.com.br 